\title{
Transversely Isotropic Constitutive Model for Extraocular Muscle Incorporating the Force-Length-Activation Relationship and Weaving Tendon
}

\author{
Asuka Hatano, ,\# Sander Schutte ${ }^{* *}$
}

\begin{abstract}
We simulated the three-dimensional contraction of the lateral rectus muscle using the finite element method. We proposed a hyperelastic, transversely isotropic constitutive model for extraocular muscle, which incorporates the force-lengthactivation relationship and the effect of changes in fraction of interweaving tendon. The model successfully reproduced the experimental force-length-activation relationship when applied to a unit cube for validation. We also propose a method to incorporate the effect of tendon weaving using the tendon fraction. The electrical potential method was applied to define the fiber direction and tendon fraction in muscle geometry derived from magnetic resonance imaging. Finally, the model was applied to a forced duction test of the lateral rectus muscle. Although our model requires further validation, the proposed constitutive model and definition of geometrical information are a step toward future medical application by facilitating three-dimensional modeling with improved accuracy.
\end{abstract}

Keywords: extraocular muscle, finite element method, constitutive equation.

Adv Biomed Eng. 4: pp. 105-111, 2015.

\section{Introduction}

The success rate of strabismus surgery is less than $20 \%$, and some patients require further surgery because of undesirable deviations in ocular alignment [1]. A possible reason for the low success rate is inaccurate estimation of biomechanical consequences due to individual variation. To better understand the complex three-dimensional (3D) extraocular muscle system, mathematical models of orbital biomechanics have long been studied. Robinson et al. [2] constructed the first quantitative model representing the mechanical state of six extraocular muscles to estimate the correction by strabismus surgery. Since then, improved extraocular muscle models have been reported, such as the SQUINT model [3], the graphical user interface gaze mechanics simulator Orbit $^{\mathrm{TM}}$ [4], and the interactive environment for strabismus surgery SEE++ [5]. In most of these models, the anatomy of the extraocular system is simplified, with the extraocular muscle represented by a string or a set of strands, and the surrounding materials as constraints on muscle paths. Although the models predict the general movement and consequence of the extraocular system, this simplification limits the models' ability in three ways. The first is insufficient consideration of the 3D interaction of muscle with surrounding material and the $3 \mathrm{D}$ behavior of the material itself (such as shear or bulk modulus). These 3D characteristics are partially addressed by adding constraints to the string model. However, this leads to the second limitation: the model requires many assumptions and the parameters do not necessarily have physical

Received on August 22, 2014; revised on January 19, 2015; accepted on April 2, 2015.

* Department of Mechanical Engineering, Graduate School of Engineering, The University of Tokyo, Tokyo, Japan.

*** Blinq Systems B.V., Delft, the Netherlands.

\# 7-3-1 Hongo, Bunkyo-ku, Tokyo 113-8656, Japan.

E-mail: hatano@fml.t.u-tokyo.ac.jp significance. Finally, the estimation of parameters for a different geometry is laborious and especially difficult in the absence of parameters having physical significance. These limitations hamper application of the models for medical purposes, because they do not allow better estimation of surgery by customizing the model.

The finite element method can be employed to overcome the above problems, because it requires fewer assumptions and retains the morphology better, and its power to reproduce the highly nonlinear 3D dynamic movement of an anisotropic muscle has been demonstrated. The method is widely used in analyzing the mechanics of skeletal muscle [6-9] and cardiac muscle [10-18]. However, there are few reports of applications of the finite element method to the mechanics of extraocular muscle, which differs in morphology and mechanical properties from skeletal and cardiac muscles. Schutte et al. [19] constructed a finite element extraocular model, in which the geometry was derived from magnetic resonance images (MRI) of the orbit structure, and the model contained the rectus muscles, fat, eyeball and socket finely meshed. However, the applied mechanical properties ignored anisotropy, the force-length relationship and the existence of tendon.

This paper complements the previous work of Schutte et al. [19] by improving mechanical representation. We propose a transversely isotropic constitutive model representing the activation-force-length relationship of extraocular muscle. The effect of tendon, which is woven into the muscle and occupies a certain fraction of the muscle volume, is also incorporated, assuming it to be a fiber reinforced composite. We also present a process to define the fiber and tendon distribution appropriately for the extraocular muscle geometry. The model was applied to the lateral rectus muscle. Finally, the proposed model was validated in a forced duction test.

\section{Method}

In previous work carried out by Schutte et al. [19], the extraocular 
geometry was obtained from frontal MRI scans of a healthy female gazing ahead. The bony orbit, muscles and eyeball were segmented and finely meshed using tetrahedral elements. This previous study had several limitations: the given active stress fiber direction was rectilinear and coarsely followed the shape outline, the existence of tendon and the force-length relationship was ignored, and the material property was modeled as isotropic despite the presence of strong anisotropy. In this study, modeling of the mechanical properties was improved.

\subsection{Constitutive Model for Extraocular Muscle}

Blemker et al. [20] proposed a transversely isotropic hyperelastic constitutive model for skeletal muscle using new strain invariants introduced by Criscione et al. [21], which have direct physical interpretations for transverse isotropic material. We adopt the same approach because of its superiority in modeling transversely isotropic material (see Discussion). The material constitutive behavior is characterized by three invariants of the right CauchyGreen strain tensor $\boldsymbol{C}$; namely, the fiber-directional stretch $(\lambda)$, fiber-directional shear $\left(\beta_{1}\right)$, and cross-fiber shear $\left(\beta_{2}\right)$ :

$$
\begin{aligned}
& \lambda=\sqrt{\tilde{I}_{4}}, \\
& \beta_{1}=\sqrt{\frac{\tilde{I}_{5}}{\tilde{I}_{4}^{2}}-1,} \\
& \beta_{2}=\cosh ^{-1}\left(\frac{\tilde{I}_{1} \tilde{I}_{4}-\tilde{I}_{5}}{2 \sqrt{\tilde{I}_{4}}}\right),
\end{aligned}
$$

where $\tilde{I}_{j}$ is the $\mathrm{j}^{\text {th }}$ reduced invariant of $\boldsymbol{C}$. (See Fig. 2 of [22] for the physical meaning of these invariants.) Using the above invariants, the strain energy function for extraocular muscle is expressed as

$$
W=W_{1}\left(\beta_{1}\right)+W_{2}\left(\beta_{2}\right)+W_{3}(\lambda, \theta)+W_{v}\left(I_{3}\right)
$$

where $W_{1}$ is the fiber-directional shear potential; $W_{2}$ is the cross-fiber shear potential; $W_{3}$ is the fiber-directional tensile potential; $W_{\mathrm{v}}$ is the volumetric potential; and $\theta$ is the gaze effort that corresponds to activation of extraocular muscle. Only $W_{3}$ depends on gaze effort. $W_{3}$ comprises active and passive tension, whereas the other variables are purely passive. We assume that each component of shear stress is linear and solely depends on the corresponding shear strain invariant. Therefore, $W_{1}$ and $W_{2}$ are simply expressed as

$$
W_{1}=\frac{1}{2} G_{1} \beta_{1}^{2}, W_{2}=\frac{1}{2} G_{2} \beta_{2}^{2},
$$

where $G_{1}$ and $G_{2}$ are the along-fiber and cross-fiber shear moduli respectively. Regarding fiber direction, we assume that fiber-directional conventional stress $f(\lambda, \theta)$ is a function of length (stretch ratio, $\lambda)$ and gaze effort $(\theta)$. $W_{3}$ follows the relationship

$$
\frac{\partial W_{3}}{\partial \lambda}=f(\lambda, \theta) \text {. }
$$

Accordingly, the second Piola-Kirchhoff stress S is expressed as

$$
S_{i j}=2 G_{1} \beta_{1} \frac{\partial \beta_{1}}{\partial C_{i j}}+2 G_{2} \beta_{2} \frac{\partial \beta_{2}}{\partial C_{i j}}+f(\lambda, \theta) N_{i} N_{j}-p C_{i j}^{-1},
$$

where $N$ is a normal vector in the fiber direction and $p$ is pressure. The above equation defines the constitutive relationship between stress and strain, as long as $G_{1}, G_{2}$ and $f(\lambda, \theta)$ are given as described in the following section.

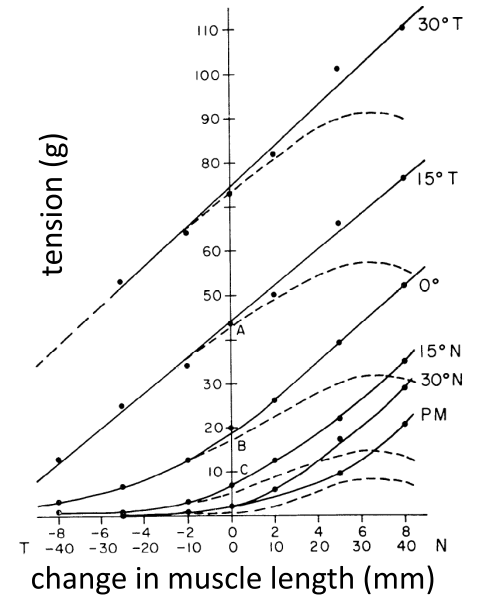

Fig. 1 Experimental force-length relationship obtained by Robinson et al. [2] (reproduced with permission from the American Physiological Society). The PM line shows passive muscle tension. The other solid lines show the total tension, and broken lines show the active tension at various gaze efforts. $\mathrm{T}$ and $\mathrm{N}$ denote temporal and nasal directions, respectively (see Fig. 2).

\subsection{Force-Length-Gaze Effort Relationship of Extraocular Muscle}

Robinson et al. [2] measured the isometric tensions of lateral rectus muscles at different gaze efforts during strabismus surgery (Fig. 1, reproduced from [2]). After the lateral and medial recti were detached, the isometric muscle tension was recorded using a strain gauge attached to the lateral rectus, while the patient was instructed to make saccadic fixation changes from the primary position to angles of $\pm 15 \pm 30 \mathrm{deg}$. Hereinafter, the temporal direction is defined as positive $\theta$. We convert the force-length relationship of the whole lateral rectus to the stress-strain relationship of muscle tissue (without tendon) considering the rectus to have a cross-sectional area of $20 \mathrm{~mm}^{2}$ [23] and effective length of $40 \mathrm{~mm}$ by subtracting the length of the mainly tendon part of the rectus, assuming that tendon is far stiffer than muscle.

To express this stress-strain relationship of extraocular muscle tissue, we employ an approach similar to that used for skeletal muscle $[20,24]$. Conventional stress $f(\lambda, \theta)$ is expressed as the sum of the passive force $f_{p}(\lambda)$ (corresponding to the curve PM in Fig. 1) and active force $f_{a}(\lambda, \theta)$ (corresponding to the dotted lines). We adopted the functional form of $f_{p}$ from skeletal muscle [22]. With regard to $f_{\mathrm{a}}$, linear dependence on activation assumed for skeletal muscle is not appropriate for extraocular muscle, and we thus propose the dependence in the following manner. First, we assume $f_{a}(\lambda, \theta)=a_{1} \exp \left\{-a_{2}\left(\lambda-\lambda_{\text {opt }}\right)^{2}\right\}$, approximated from the human extraocular force-length relationship measured by Robinson et al. [2] (Fig. 1), where $a_{1}$ and $a_{2}$ are second-order functions of $\theta$, and $\lambda_{\text {opt }}$ is the optimal stretch that exerts the maximum force. At each gaze effort, $a_{1}$ corresponds to the maximal active force and $a_{2}$ determines the curvature, which can be obtained from the $\lambda=0$ intercept. Table 1 shows the maximum forces and intercepts of active forces (broken line) at $\theta=30^{\circ} \mathrm{N}, 15^{\circ} \mathrm{N}$, $0^{\circ}, 15^{\circ} \mathrm{T}$, and $30^{\circ} \mathrm{T}$ obtained from the graphs shown in Fig. 1. We then assume $a_{1}$ and $a_{2}$ to be second-order functions of gaze effort and fit coefficients of $a_{i j}$ in Eqs. 11 and 12 using the values in 
Table 1 Intercept and maximum active force at each gaze effort read from the graph of Robinson et al. [2] and used to fit model parameters.

\begin{tabular}{rrr}
\hline \hline gaze effort $(\mathbf{d e g})$ & intercept [g] & maximum [g] \\
\hline$-30\left(30^{\circ} \mathrm{N}\right)$ & 1 & 8 \\
$-15\left(15^{\circ} \mathrm{N}\right)$ & 4 & 15 \\
$0 \quad\left(0^{\circ}\right)$ & 16 & 32 \\
$15\left(15^{\circ} \mathrm{T}\right)$ & 42 & 56 \\
$30\left(30^{\circ} \mathrm{T}\right)$ & 72 & 90 \\
\hline \hline
\end{tabular}

Table 2 Model parameters.

\begin{tabular}{lrl|lrl}
\hline \hline parameter & value & unit & parameter & value & unit \\
\hline$G_{1}^{\text {muscle }}$ & 50 & $\mathrm{MPa}$ & $a_{10}$ & 31.5 & - \\
$G_{2}^{\text {muscle }}$ & 50 & $\mathrm{MPa}$ & $a_{11}$ & 1.37 & - \\
$b_{1}^{\text {muscle }}$ & 1.0 & - & $a_{12}$ & 0.02 & - \\
$G_{2}^{\text {muscle }}$ & 12 & - & $a_{20}$ & 26.6 & - \\
$G_{1}^{\text {tendon }}$ & 500 & $\mathrm{MPa}$ & $a_{21}$ & -1.2 & - \\
$G_{2}^{\text {tendon }}$ & 500 & $\mathrm{MPa}$ & $a_{22}$ & 0.02 & - \\
$b_{1}^{\text {tendon }}$ & $2.7 \times 10^{3}$ & - & $\lambda_{o p t}$ & $1.16 \mathrm{~mm}$ \\
$b_{2}^{\text {tendon }}$ & 46.4 & - & $\lambda_{m p}$ & $0.93 \mathrm{~mm}$ \\
\hline \hline
\end{tabular}

Table 1, employing the least-squares method. We finally obtain the equations

$$
\begin{aligned}
& f(\lambda, \theta)=f_{p}(\lambda)+f_{a}(\lambda, \theta), \\
& f_{p}(\lambda)=\left\{\begin{array}{cc}
0 & \lambda<\lambda_{m p} \\
b_{1}\left(e^{b_{2}\left(\lambda-\lambda_{m p}\right)}-1\right) & \text { else }
\end{array}\right. \\
& f_{a}(\lambda, \theta)=a_{1} e^{-a_{2}\left(\lambda-\lambda_{\text {opt }}\right)^{2}}, \\
& a_{1}=a_{12} \theta^{2}+a_{11} \theta+a_{10}, \\
& a_{2}=a_{22} \theta^{2}+a_{21} \theta+a_{20},
\end{aligned}
$$

where $\lambda_{m p}$ is the minimal stretch that exerts a passive force, with the parameter values shown in Table 2.

\subsection{Description of Tendon}

Anatomically, no strict separation of muscle and tendon has been found in extraocular muscles. Collagen fibers interweave with muscle fibers. There is $100 \%$ collagen (tendon) anteriorly (to $5 \mathrm{~mm}$ ) and mainly muscle fibers posteriorly. To describe the gradual change in material properties, we define the tendon fraction $\gamma$ $(0 \leq \gamma \leq 1)$, and assume that the fiber direction of tendon runs solely parallel to that of muscles. Under this assumption, muscle with weaving tendon can be considered as unidirectional fiber-reinforced composite. We adopt Halpin-Tsai equations [25] to describe the reduced moduli from the moduli of fiber (tendon) and matrix (muscle). Regarding the fiber direction, the parallel spring model can be used when the two constituents have the same Poisson ratio; in this case, both are incompressible.

$$
f^{\text {weave }}=\gamma f^{\text {tendon }}+(1-\gamma) f^{\text {muscle }} \text {. }
$$

For the shear modulus,
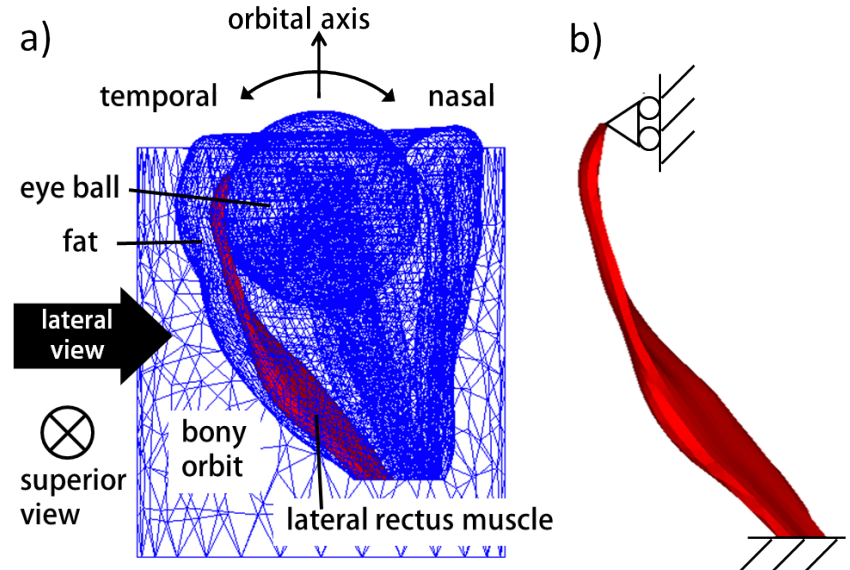

Fig. 2 a) Finite element mesh of the lateral rectus muscle shown with a mesh structure of the whole extraocular system. b) The provisory boundary conditions given in the calculations.

$$
G_{i}^{\text {weave }}=\frac{1+\zeta_{i} \eta_{i} \gamma}{1-\eta_{i} \gamma} G_{i}^{\text {muscle }}, \eta_{i}=\frac{G_{i}^{\text {tendon }}-G_{i}^{\text {muscle }}}{G_{i}^{\text {tendon }}+\zeta_{i} G_{i}^{\text {muscle }}} .
$$

In the case of fiber directional shear $(i=1), \zeta_{1}=1$. In the case of cross-fiber shear $(i=2)$ though, $\zeta_{2}$ depends on the ratio of the plane strain bulk modulus to the transverse shear modulus of matrix constituents (muscle). We approximate $\zeta_{2} \cong 1$ because the uncertainty in $\gamma$ exceeds the effect of $\zeta_{2}$. Therefore,

$$
G_{i}^{\text {weave }}=\frac{(1+\gamma) G_{i}^{\text {tendon }}+(1-\gamma) G_{i}^{\text {muscle }}}{(1-\gamma) G_{i}^{\text {tendon }}+(1+\gamma) G_{i}^{\text {muscle }}} G_{i}^{\text {muscle }} .
$$

Applying the above $f^{\text {weave }}$ and $G_{i}^{\text {weave }}$ to Eqs. 1 and 7, a gradual change in muscle property is obtained with thinning of the weaving tendon.

\subsection{Distribution of Fiber Orientation and Tendon}

We use the tetrahedral mesh of lateral rectus muscle previously obtained from MRI images and meshed using the Patran software (Fig. 2) [19]. We choose the lateral rectus muscle to simplify the problem. Because obtaining the fiber direction and tendon fraction simultaneously with the geometry for the same MRI measurement is difficult, it is practical to add the information afterwards according to anatomical knowledge. We define the fiber direction by applying the electrical potential method, which is a mapping method proposed by Hisada et al. [26]. Using this method, the fiber direction is mapped along the simulated electrical flux virtually applied between the edges of the lateral rectus mesh. All the muscle paths are supposed to be continuous along the surface and do not cross each other, which is analogous to the path of electrical flux when electrical potential is applied between the edges of muscle fibers. Electrical potential $\phi$ is calculated by solving the Laplace equation

$$
\begin{aligned}
& \nabla^{2} \phi=0, \\
& \left\{\begin{array}{ccc}
\text { on } & S_{\text {edge } 0} & \phi=0 \\
\text { on } & S_{\text {edge } 1} & \phi=1 \\
\text { on } & S_{\text {other }} & \boldsymbol{n}_{s} \cdot \nabla \phi=0
\end{array},\right.
\end{aligned}
$$

and the fiber direction $N$ is defined as

$$
N=\frac{\nabla \phi}{|\nabla \phi|},
$$

where $S_{\text {edge0 }}$ and $S_{\text {edgel }}$ are the surfaces where muscle fiber starts 
and ends, and $S_{\text {other }}$ is a surface other than $S_{\text {edge0 }}$ or $S_{\text {edge1 }}$. $\boldsymbol{n}_{\mathrm{s}}$ denotes a vector normal to the surface. The Neumann boundary condition ensures that the fiber does not extend from the mesh surface other than at the two edges. In applying the mesh of the lateral rectus muscle, we set the surface of the base for $S_{\text {edge } 0}$ and the insertion for $S_{\text {edgel }}$.

In defining the tendon fraction, we use the potential $\phi$ obtained above, which can be regarded as the normalized (though the scale is exponential) position from the insertion to the base. We set the tendon fraction $\gamma$ as

$$
\left\{\begin{array}{lcc}
\gamma= & 1.0 & 0.70 \leq \phi<1.0 \\
\gamma=(0.7-\phi) / 0.45 & 0.25 \leq \phi<0.70 \\
\gamma= & 0 & \phi \leq 0.25
\end{array} .\right.
$$

Here, the range $0.70 \leq \phi<1.0$ corresponds to a distance within $\sim 5 \mathrm{~mm}$ from the insertion, and $\phi=1.0$ corresponds to a distance $\sim 20 \mathrm{~mm}$ from the insertion.

\subsection{Boundary Conditions and Calculations}

We first performed calculations to validate the proposed constitutive model for muscle tissue (without tendon) and our simulation programs. A unit cube $(1 \mathrm{~mm} \times 1 \mathrm{~mm} \times 1 \mathrm{~mm})$ consisted of 48 tetrahedral elements with purely axial fiber orientation. Six calculations were performed, comprising one passive calculation without the active tension and five calculations including the active tension fixed at $-30,-15,0,15$ and 30 degrees. Axial displacement was fixed at zero on one of the surfaces normal to the fiber axis, and was varied from -0.2 to $0.2 \mathrm{~mm}$ on the other. On other surfaces parallel to the fiber axis, stress was set to zero.

In calculations using the fiber-tendon composite constitutive equation applied to a mesh of the lateral rectus muscle with calculated fiber and tendon distribution (LR model), the displacement of all nodes on the basement was fixed, insertion nodes were fixed along the line parallel to the orbital axis as shown in Fig. 2, and stress was set to zero on other surfaces. In the forced duction test, loading in the direction of the orbital axis at the insertion was increased incrementally until the length of the displacement at the insertion reached $8 \mathrm{~mm}$.

The type of tetrahedral element was $5 / 4 \mathrm{c}$. All codes (including solver and finite element integration) were written in Fortran, and the computation was performed using an Intel Xeon central processing unit $(3.2 \mathrm{GHz})$.

\section{Results}

\subsection{Validation of the Proposed Constitutive Model for Mus- cle Tissue}

For validation of the proposed constitutive model for muscle tissue and our finite element method code, we first simulated the fiber directional tension test with a unit cube consisting of 48 tetrahedral elements. The simulated force-length relationship for varying gaze efforts of $-30,-15,0,15$, and 30 degrees is plotted in Fig. 3. We verified that the simulated relationship is consistent with previous experimental results as shown in Fig. 1 (reproduced from [2]).

\subsection{Distribution of Fiber and Tendon}

Figure 4 shows the calculated fiber direction $(a, b)$, and fraction of tendon $(c, d)$ of the lateral rectus muscle in superior $(a, c)$ and lateral views (b, d). Each short line segment indicates the fiber direction of a tetrahedral element $(a, b)$. Smooth changes in fiber

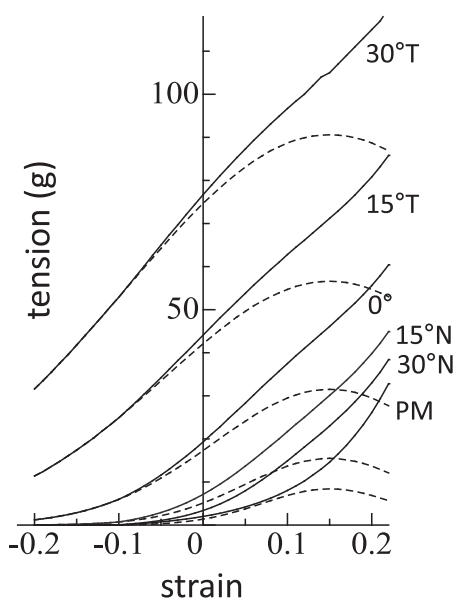

Fig. 3 Simulated force-length relationship obtained by applying the proposed muscle tissue constitutive equation on the unit cube. Solid lines show the total tension, and broken lines show the active component.
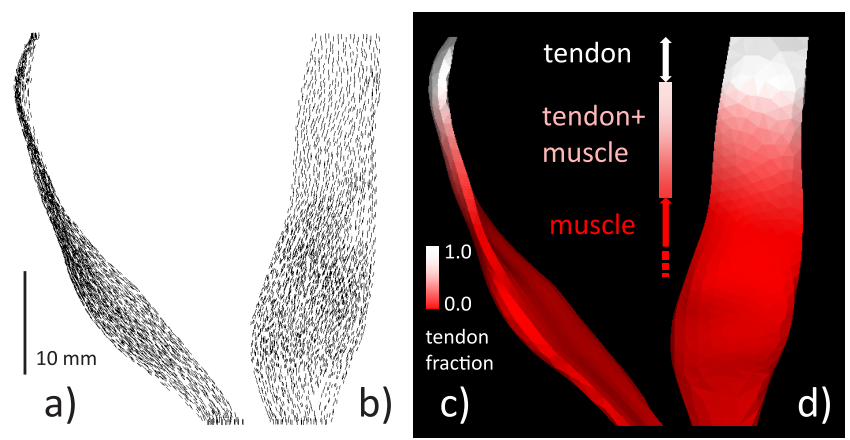

Fig. 4 Calculated distribution of fiber ( $a$ and b) and tendon ( $c$ and d) of the lateral rectus muscle, in superior view (a and c) and lateral view ( $b$ and $d)$.

direction along the outline of the muscle belly were observed. In $\mathrm{c}$ and $\mathrm{d}$, muscle is colored red and tendon white, and the mixed region is a gradation of red and white according to the fractions of muscle and tendon.

\subsection{Free Contraction with the LR Model}

Applying the above fiber direction, tendon fraction and muscle-tendon composite constitutive model, we simulated the deformation upon changing gaze effort without loading. Gaze effort was increased incrementally by 5 degrees from -30 to +30 degrees. Figure 5 shows the deformation, color coded to indicate the distribution of the fiber-directional stretch ratio. Upon increasing the gaze effort, the muscle gradually contracted, and the insertion was displaced $9 \mathrm{~mm}$. The stretch ratio was mostly within 1 (shortening) due to the absence of a load, although small local lengthening (stretch ratio less than 1.04) was observed under a large gaze effort, with a boundary condition and geometrical factor. For each gaze effort, the stretch ratio was reduced toward the direction of insertion. This was due to the increasing fraction of tendon, which is stiffer than muscle and does not actively generate force. Around the rectus muscle belly, the stretch ratio was relatively homogeneous, although the periphery of the muscle 


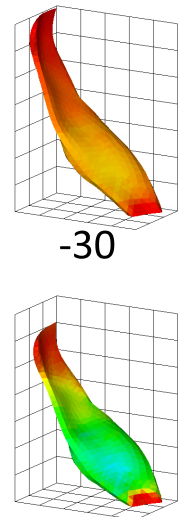

10

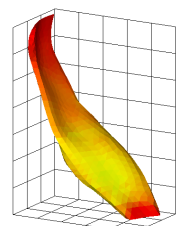

$-20$

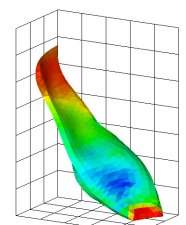

20

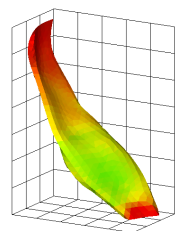

$-10$

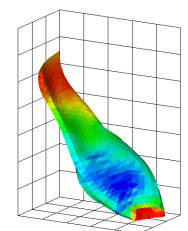

30

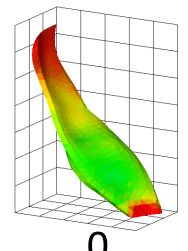

1.0

0.6 stretch ratio

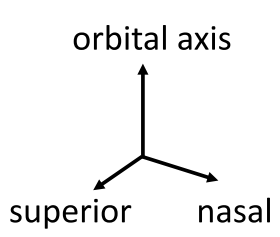

gaze effort (deg)
Fig. 5 Deformations under unloaded contraction. The distribution of the stress stretch ratio is indicated by color, for each gaze effort (deg). Grid size is $5 \mathrm{~mm}$.

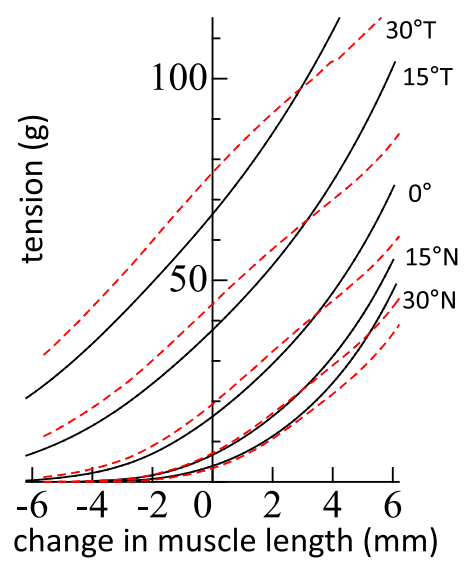

Fig. 6 Simulated force-length relationship of the LR model (applying the proposed muscle and tendon composite representation to the lateral rectus muscle mesh with calculated fiber and tendon distribution). Solid lines show the total tension and broken lines the unit cube tension-length relationship (solid line in Fig. 3) for comparison.

was slightly elongated compared to the center because of isovolumic contraction. We observed that the LR model skewed to rotate the eyeball in a superior direction with a shift in gaze effort in the temporal direction.

\subsection{Forced duction Test using the LR Model}

Next, a fiber-directional forced-duction test was simulated for varying gaze efforts of $30,15,0,-15$, and -30 degrees. The simulated force-length relations of the whole lateral rectus muscle at various gaze efforts are shown by solid lines in Fig. 6. The change in length of the LR model was calculated as displacement of the insertion, with the original position assumed at a point when passive muscle exerts a force of $2 \mathrm{~g}$. Dotted lines show the unit-cube tension-length relationship for comparison, assuming effective muscle length in this model as $30 \mathrm{~mm}$. When gaze effort was negative, the simulated force-length relationship in the range of small length change was in good agreement with the unit cube tension-length relationship. However, we noted disagreement when gaze effort was positive. In particular, the force-length relationship was nonlinear when the change in length was large.

\section{Discussion}

The objective of this paper is to present a constitutive model and scheme with which to define geometric information of extraocular muscle for 3D finite element analysis of orbital biomechanics. This section describes the new features of our model and discusses the prospects for future medical applications.

\subsection{Constitutive model for extraocular muscle}

We propose a hyperelastic, constitutive model for extraocular muscle tissue. Our model successfully reproduced the forcelength-activation (gaze effort) relationship of extraocular muscle when applied to a unit cube for validation. Additionally, we propose a method to incorporate the effect of tendon weaving using the tendon fraction. These features were realized by applying the new invariants $\left(\lambda, \beta_{1}\right.$, and $\left.\beta_{2}\right)$ proposed by Criscione et al. [21], which have direct physical interpretations, allowing explicit and independent representation of the material in each direction.

\subsection{Definition of fiber and tendon}

In finite element analysis of transversely isotropic material, a fiber direction vector is needed as input for each element in the mesh. The importance of obtaining the proper fiber orientation upon muscle simulation has been recognized. Tang et al. [9] showed that the fiber direction affects the deformation and strain distribution in skeletal muscle simulations. Washio et al. [27] optimized heart fiber directions and obtained an ejection volume that was more than $10 \%$ better than that of the initial mapping of fiber directions.

Although fiber orientation is measurable, many measurements are required. Therefore, in finite element analysis, the fiber direction is often mapped according to anatomical knowledge, such as the outline of the fusiform [9], polynomial fits [8], or interpolated splines.

We applied the electrical potential method proposed by Hisada et al. [26], which is a modeling device used to project orientation-related characteristics (in our case, the fiber direction itself) according to the electric current orientation obtained by virtually electrifying a muscle section. We only needed information of where fibers start and end as the boundary condition for virtual electrifying, and these are already defined as the insertion and basement. The electrical current-based orientation obtained is consistently smooth and continuous along the outline. In addition, the process can be automated for future applications.

The virtual electrical potential was subsequently used to define the continuous thinning of the tendon. Without the tendon, the thinner part of the muscle near the insertion was unrealistically elongated under loading (data not shown). In contrast, when the bundle was partitioned into tendon and muscle, the tendon barely stretched whereas the muscle portion at the boundary with the tendon had large localized strain. Our proper definition of tendon based on anatomy successfully avoids strong nonlinearity caused by morphology. However, to our knowledge, experimental measurements of material responses to change in fraction of weaving tendon have not been reported. Our assumptions for the constitutive model and distribution of tendon require experimen- 
tal verification.

\subsection{Limitations and future directions}

As the first limitation of our model, in the forced duction test using the mesh structure of the lateral rectus muscle, we observed disagreement of the force-length relationship compared to the reference experimental data. This disagreement is inevitable, because we used the experimental results for the whole-rectus tension test (which reflect morphology and material distributions) to define muscle segment properties. The disagreement might be avoided if we fit the model parameters by comparing the simulation curve of the LR model (applying the composite constitutive equation on the whole rectus with distributed tendon and fiber) with the experimental curve. However, this approach is not possible, because 1) it has a huge computational cost, 2) the proposed parameters would nevertheless be unreliable owing to uncertainty in material properties and the distribution of tendon, and 3) it defeats the benefit of using new invariants to obtain direct and explicit expression and applying the results of an experimental forced duction test to a constitutive model. For fundamental solution, we need experimental material data for individual muscle segments, because the material property is not homogeneous throughout the muscle. In particular, material properties are affected by the existence of tendon. After obtaining comprehensive experimental data, our model can be improved and validated further.

Second, we observed that the lateral rectus muscle skewed to rotate the eyeball in a superior direction. Because we could not find a reference to the lateral rectus exerting a force to rotate the eyeball in the superior direction, we suppose that this movement is incorrectly caused by oversimplified boundary conditions. The basement should be connected to the socket more posteriorly, and the surrounding fat should provide resistance against bending. Integrated simulation including interactions with the whole extraocular system, such as displacing the orbital fat and making contact with the eye socket, would provide medically significant insights.

The important feature of the 3D finite element analysis is the ability to evaluate the effect of morphological differences and interactions with the surrounding materials. This is important when taking individual differences into account. Our proposal of a constitutive model and definition of geometrical information is a step toward future medical application through facilitating modeling with improved accuracy.

\section{Acknowledgements}

This research was supported by JSPS Grant-in-Aid for Research Activity Start-up Grant number 25882010, and by the Japan Student Services Organization through its "Interdisciplinary Global Mechanical Engineering Education” program. The authors would like to thank Hideaki Murayama, Shinsuke Sakai, Riender Happee and Tetsuo Tomiyama for their support.

\section{Conflicts of interest}

We have no conflicts of interest relationship with any companies or commercial organizations based on the definition of Japanese Society of Medical and Biological Engineering.

\section{References}

1. Zhang MS, Hutchinson AK, Drack AV, Cleveland J, Lambert SR:
Improved ocular alignment with adjustable sutures in adults undergoing strabismus surgery. Ophthalmology. 119(2), pp. 396402, 2012.

2. Robinson DA, O'Meara DM, Scott AB, Collins CC: Mechanical components of human eye movements. J Appl Physiol. 26(5), pp. 548-553, 1969.

3. Miller JM, Robinson DA: A model of the mechanics of binocular alignment. Comput Biomed Res. 17(5), pp. 436-470, 1984.

4. Miller JM: Understanding and misunderstanding extraocular muscle pulleys. J Vis. 7(11), pp. 1-15, 2007.

5. Haslwanter T, Buchberger M, Kaltofen T, Hoerantner R, Priglinger S: SEE++: a biomechanical model of the oculomotor plant. Ann N Y Acad Sci. 1039, pp. 9-14, 2005.

6. Martins JAC, Pires EB, Salvado R, Dinis PB: A numerical model of passive and active behavior of skeletal muscles. Comput Methods Appl Mech Eng. 151(3-4), pp. 419-433, 1998.

7. Johansson T, Meier P, Blickhan R: A finite-element model for the mechanical analysis of skeletal muscles. J Theor Biol. 206(1), pp. 131-149, 2000.

8. Oomens CWJ, Maenhout M, van Oijen CH, Drost MR, Baaijens FP: Finite element modelling of contracting skeletal muscle. Philos Trans R Soc Lond B Biol Sci. 358(1437), pp. 1453-1460, 2003.

9. Tang CY, Zhang G, Tsui CP: A 3D skeletal muscle model coupled with active contraction of muscle fibres and hyperelastic behaviour. J Biomech. 42(7), pp. 865-872, 2009.

10. Chuong CJ, Fung YC: Three-Dimensional Stress Distribution in Arteries. J Biomech Eng. 105(3), pp. 268-274, 1983.

11. Humphrey JD, Yin FCP: On constitutive relations and finite deformations of passive cardiac tissue: I. a pseudostrain-energy function. J Biomech Eng. 109(4), pp. 298-304, 1987.

12. Humphrey JD, Strumpf RK, Yin FCP: Determination of a constitutive relation for passive myocardium: I. a new functional form. J Biomech Eng. 112(3), pp. 333-339, 1990.

13. Nash M, Hunter P: Computational mechanics of the heart. J Elasticity. 61(1), pp. 113-141, 2000.

14. Yin FCP, Strumpf RK, Chew PH, Zeger SL: Quantification of the mechanical properties of noncontracting canine myocardium under simultaneous biaxial loading. J Biomech. 20(6), pp. 577-589, 1987.

15. Lin DHS, Yin FCP: A Multiaxial constitutive law for mammalian left ventricular myocardium in steady-state barium contracture or tetanus. J Biomech Eng. 120(4), pp. 504-517, 1998.

16. Watanabe H, Sugiura S, Kafuku H, Hisada T: Multiphysics simulation of left vntricular filling dynamics using fluid-structure interaction finite element method. Biophy J. 87(3), pp. 20742085, 2004.

17. Hatano A, Okada J-i, Washio T, Hisada T, Sugiura S: A three-dimensional simulation model of cardiomyocyte integrating excitation-contraction coupling and metabolism. Biophy J. 101(11), pp. 2601-2610, 2011.

18. Hatano A, Okada J-i, Hisada T, Sugiura S: Critical role of cardiac t-tubule system for the maintenance of contractile function revealed by a 3D integrated model of cardiomyocytes. J Biomech. 45(5), pp. 815-823, 2012.

19. Schutte S, van den Bedem SPW, van Keulen F, van der Helm FCT, Simonsz HJ: A finite-element analysis model of orbital biomechanics. Vision Res. 46(11), pp. 1724-1731, 2006.

20. Blemker SS: 3D Modeling of complex Muscle Architecture and Geometry. Stanford Universtity; 2004.

21. Criscione J, Douglas A, Hunter W: Physically based strain invariant set for materials exhibiting transversely isotropic behavior. $\mathrm{J}$ 
Mech Phys Solids. 49(4), pp. 871-897, 2001.

22. Silvia SB, Peter MP, Scott LD: A 3D model of muscle reveals the causes of nonuniform strains in the biceps brachii. J Biomech. 38(4), pp. 657-665, 2005.

23. Miller JM: Functional anatomy of normal human rectus muscles. Vision Res. 29(2), pp. 223-240, 1989.

24. Zajac FE: Muscle and tendon: properties, models, scaling, and application to biomechanics and motor control. Crit Rev Biomed Eng. 17(4), pp. 359-411, 1989.

25. Affdl JCH, Kardos JL: The Halpin-Tsai equations: a review. Polym Eng Sci. 16(5), pp. 344-352, 1976.

26. Hisada T, Kurokawa H, Oshida N, Yamamoto M, Washio T, Okada $\mathrm{J}$, et al.: Modeling device, program, computer-readable recording medium, and method of establishing correspondence. United States Patent 2012.

27. Washio T, Okada J, Sugiura S, Hisada T: Large-scale integrated model is useful for understanding heart mechanisms and developments of medical therapy. Conf Proc IEEE Eng Med Biol Soc. 2009, pp. 2347-50, 2009.

\section{Asuka Hatano}

Asuka Hatano received the B.S. degree from School of Engineering, the University of Tokyo, and the M.S. and Ph.D. degrees from Graduate School of Frontier Sciences, the university of Tokyo. She is an associate professor of Graduate School of Engineering at the University of Tokyo,

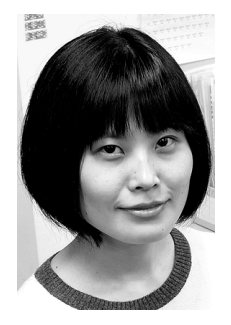
since 2013. Her research interests focus on modeling and simulation of multi-physics biological phenomena.

\section{Sander SchutTe}

Sander SchutTe received the B.Sc. degree in mechanical engineering and the M.Sc. degree in biomedical engineering. The subject of his Ph.D. thesis was the soft-tissue biomechanics of the orbit and the eye improvement of strabismus surgery. $\mathrm{He}$ is now CEO of the Dutch software company

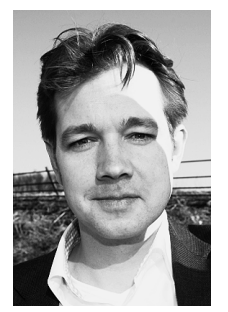
Blinq Systems. 